\title{
The interaction of linear boundary value and nonlinear functional conditions
}

\author{
by Michal FEČKAN (Bratislava)
}

\begin{abstract}
The existence of solutions is studied for certain nonlinear differential equations with both linear and nonlinear conditions.
\end{abstract}

1. Introduction. The purpose of this paper is to study the existence of solutions of problems similar to

$$
\begin{aligned}
& x^{\prime \prime \prime}=f(x, t), \\
& \left\|x^{\prime \prime}\right\|_{C^{0}}=g(x), \\
& x(0)=x(1)=0,
\end{aligned}
$$

where $f: \mathbb{R} \times[0,1] \rightarrow \mathbb{R}$ and $g: C^{0}([0,1], \mathbb{R}) \rightarrow \mathbb{R}$ are continuous. We see that (1.1) contains two types of conditions. The first is nonlinear and functional, while the second is linear and involves boundary values. Moreover, the second condition usually leads to problems at resonances.

Problems with the first type of conditions were studied in [2]. S. A. Brykalov solved the problem

$$
\begin{aligned}
& x^{\prime \prime}=f(x, t), \\
& \left\|x^{\prime}\right\|_{C^{0}}=g_{1}(x), \\
& \|x\|_{C^{0}}=g_{2}(x),
\end{aligned}
$$

where $f: \mathbb{R} \times[0,1] \rightarrow \mathbb{R}$ and $g_{1}, g_{2}: C^{0}([0,1], \mathbb{R}) \rightarrow \mathbb{R}$ are continuous. Under additional assumptions on $f, g_{1}, g_{2}$, he showed the existence of at least four solutions of the problem. Hence the paper [2] suggests a method for finding multiple solutions for nonlinear ordinary differential equations with certain nonlinear, functional conditions.

On the other hand, the theory of existence of solutions for nonlinear

1991 Mathematics Subject Classification: 46N20, 47H17, 34B15.

Key words and phrases: differential equations, linear and nonlinear conditions, Fredholm operators. 
boundary value problems at resonances is well-known [1]. The so-called Landesman-Lazer conditions play an important role in that theory.

In this paper, we try to combine these two approaches to (1.1). We shall study certain types of nonlinear ordinary differential equations with both linear boundary conditions and nonlinear functional conditions. We were motivated mainly by [2]; as far as the author knows, the literature on such problems is rather limited, and their study remains a promising subject. On the other hand, S. A. Brykalov did not consider linear boundary value conditions which may lead to problems at resonances. Moreover, in some sense his nonlinear functional conditions are more specific than ours. Our setting of the problem is more general, and it embodies a broader variety of nonlinear differential equations, due to the combination of two types of conditions.

The plan of this paper is as follows. In the next section, we study an abstract equation in Banach spaces, generalizing (1.1). Then we apply our results on that equation to ordinary differential equations similar to (1.1). Section 4 includes some remarks.

2. Abstract results. In this section, we formulate an abstract version of (1.1) in the framework of nonlinear operators in Banach spaces. We refer the reader to the next section for concrete forms of those operators and spaces. We study an abstract equation of the form

$$
L u=H(u), \quad \bar{B}(u)=D(u),
$$

where $L: X \rightarrow Y, H: Y \rightarrow Y, \bar{B}: X \rightarrow \mathbb{R}^{n}$ and $D: Y \rightarrow \mathbb{R}^{n}$ are continuous, $L$ is a linear operator, $X, Y$ are Banach spaces and $X$ is compactly embedded into $Y$. Moreover, we assume $L=L_{1} \circ L_{2}$, where $L_{1}: X_{1} \rightarrow Y$ and $L_{2}: X_{2} \rightarrow Y$ are continuous and linear, and $L_{2}$ is Fredholm of nonnegative index. Furthermore, $X_{1}, X_{2}$ are Banach spaces such that $X \subset X_{2}$ and $X_{1}, X_{2}$ are both compactly embedded into $Y$. Finally, $X \stackrel{L_{2}}{\rightarrow} X_{1} \stackrel{L_{1}}{\rightarrow} Y$ and $\bar{B}=B \circ L_{2}$, where $B: Y \rightarrow \mathbb{R}^{n}$ is continuous.

The decompositions $L=L_{1} \circ L_{2}$ and $\bar{B}=B \circ L_{2}$ express the interaction of two conditions, and allow (2.1) to be rewritten in the following form:

$$
\begin{aligned}
& \begin{cases}L_{1} v=H(u), & v \in X_{1}, \\
B(v)=D(u), & u \in Y,\end{cases} \\
& L_{2} u=v, \quad u \in X_{2}, v \in Y .
\end{aligned}
$$

Finally, we suppose $H, D$ to be bounded, i.e., $|H(\cdot)|_{Y} \leq K_{1}$ and $K_{2} \leq$ $D(\cdot) \leq K_{3}$ for some constants $K_{1}, K_{2}, K_{3}$. (The norm of $Y$ will be denoted by $|\cdot|_{Y}$, and similarly for other spaces.)

First, we study (2.2a). For this purpose, we assume the existence of a closed subset $\mathcal{A} \subset X_{1}$ with the properties 
(a) $\forall b \in Y,|b|_{Y} \leq K_{1}, \forall d \in\left[K_{2}, K_{3}\right]$ there exists a unique $v \in \mathcal{A}$ such that $L_{1} v=b$ and $B(v)=d$.

(b) The above-defined map $(b, d) \rightarrow v(b, d)$ is continuous as a map

$$
B_{K_{1}} \times\left[K_{2}, K_{3}\right] \rightarrow Y, \quad \text { where } B_{K_{1}}=\left\{\left.z \in Y|| z\right|_{Y} \leq K_{1}\right\} .
$$

Remark 2.1. Assume that the map $v$ from (a) is bounded, i.e., $|v(\cdot, \cdot)|_{X_{1}}$ $\leq M$ for a constant $M$, and $\operatorname{dim} \operatorname{ker} L_{1}<\infty, \operatorname{Im} L_{1}=Y$. Then (a) implies (b). Indeed, let $\widetilde{L}_{1}$ be a right inverse of $L_{1}$. We take $\left|b_{i}\right|_{Y} \leq K_{1}, b_{i} \rightarrow b$ in $Y, d_{i} \rightarrow d, d_{i} \in\left[K_{2}, K_{3}\right]$. Then $\left\{\widetilde{L}_{1} b_{i}-v\left(b_{i}, d_{i}\right)\right\}_{i=1}^{\infty} \subset \operatorname{ker} L_{1}$ is a bounded subset. Hence we can assume $v\left(b_{i}, d_{i}\right) \rightarrow w$ in $X_{1}$. This implies $L_{1} w=b$ and $B(w)=d$. Thus $w=v(b, d)$ and the assertion is proved.

Now we insert the map $V(u)=v(H(u), D(u))$ into $(2.2 \mathrm{~b})$ :

$$
L_{2} u=V(u), \quad u \in X_{2} .
$$

Since $L_{2}$ is Fredholm, (2.3) can be handled in the standard way [1]. So, (2.3) is equivalent to

$$
\begin{aligned}
& u_{1}=\widetilde{L}_{2} Q V\left(u_{1}+u_{2}\right), \\
& u_{2}=u_{2}+P V\left(u_{1}+u_{2}\right), \\
& u_{2} \in \operatorname{ker} L_{2}, \quad u_{1} \in \widetilde{X}_{2}, \quad \operatorname{ker} L_{2} \oplus \widetilde{X}_{2}=X_{2}, \\
& Q: Y \rightarrow \operatorname{Im} L_{2}, \quad Q+P=I, \\
& Q \text { is a continuous projection, } \\
& \widetilde{L}_{2}=\left(L_{2} / \widetilde{X}_{2}\right)^{-1} .
\end{aligned}
$$

Summing up, we obtain

Proposition 2.2. Under the above conditions (a), (b), the equation (2.1) can be rewritten in the form (2.4).

Remark 2.3. The set $\mathcal{A}$ has been introduced for the same purpose as in [2]. By choosing another set $\mathcal{A}$, we can show the existence of multiple solutions of (2.1).

Now we assume

$$
\begin{aligned}
& \lim _{r \rightarrow \infty} H\left(r u_{2}+u_{1}\right)=H_{\infty}\left(u_{2}\right), \\
& \lim _{r \rightarrow \infty} D\left(r u_{2}+u_{1}\right)=D_{\infty}\left(u_{2}\right),
\end{aligned}
$$

uniformly in $u_{1}$ from bounded subsets and $u_{2} \in \operatorname{ker} L_{2},\left|u_{2}\right|_{X_{2}}=1$.

Moreover, suppose the maps

$H_{\infty}: S_{1}=\left\{\left.u \in \operatorname{ker} L_{2}|| u\right|_{X_{2}}=1\right\} \rightarrow Y$ and $D_{\infty}: S_{1} \rightarrow \mathbb{R}^{n}$ are continuous. 
Of course, (P1-3) imply the boundedness of $H, D$, respectively, and $\left|H_{\infty}(\cdot)\right|_{Y} \leq K_{1}, D_{\infty}(\cdot) \in\left[K_{2}, K_{3}\right]$. Hence we can define $V_{\infty}=v\left(H_{\infty}, D_{\infty}\right)$. It is clear that

$$
\lim _{r \rightarrow \infty} V\left(r u_{2}+u_{1}\right)=V_{\infty}\left(u_{2}\right)
$$

uniformly in $u_{1}$ from bounded subsets and $u_{2} \in S_{1}$. Now applying [1, (5.4.32) Theorem] we obtain

THEOREM 2.4. Let the conditions (a), (b), and (P1-3) be satisfied. Assume that $P V_{\infty}(a) \neq 0, \forall a \in S_{1}$, and that the stable homotopy class of $\eta(a)=P V_{\infty}(a) /\left|P V_{\infty}(a)\right|_{Y}, \eta: S_{1} \simeq S^{m-1} \rightarrow S^{m^{*}-1}$, is nontrivial, where $m=\operatorname{dim} \operatorname{ker} L_{2}$ and $m^{*}=\operatorname{codim} \operatorname{Im} L_{2}$. Then the equation (2.1) has a solution.

Now we shall study a special case of (2.1). We make the following assumption:

(H1) $\operatorname{Im} L_{1}=Y, \operatorname{ker} L_{1}=\mathbb{R} v_{0}, B: Y \rightarrow[\widetilde{d}, \infty) \subset \mathbb{R}, \tilde{d}>-\infty$, is continuous and strictly convex, and $B^{-1}([a, b])$ is bounded for any $-\infty<a \leq b<\infty$.

We put

$$
\begin{aligned}
& \mathcal{A}_{+}=\left\{v \in X_{1} \mid B\left(v+c v_{0}\right) \geq B(v), \forall c>0\right\}, \\
& \mathcal{A}_{-}=\left\{v \in X_{1} \mid B\left(v+c v_{0}\right) \geq B(v), \forall c<0\right\} .
\end{aligned}
$$

We note that $\tau: c \rightarrow B\left(v+c v_{0}\right)$ is a strictly convex real function such that $\tau(\mathbb{R}) \subset[\widetilde{d}, \infty)$ and $\tau^{-1}([a, b])$ is bounded for any $-\infty<a \leq b<\infty$. Hence there is a unique $c_{0} \in \mathbb{R}$ such that $c_{0}$ minimizes $\tau$, and $\tau$ is increasing for $c>c_{0}$ and decreasing for $c<c_{0}$. Thus $v+c_{0} v_{0} \in \mathcal{A}_{+} \cap \mathcal{A}_{-}$and $\mathcal{A}_{-} \cup \mathcal{A}_{+}=X_{1}$.

Now we suppose

(H2) $\quad D: Y \rightarrow \mathbb{R}$ and $H: Y \rightarrow Y$ are continuous and $|H(\cdot)|_{Y} \leq K_{1}, K_{2} \leq$ $D(\cdot) \leq K_{3}$ for some constants $K_{1}, K_{2}, K_{3}$.

(H3) For any $v \in \mathcal{A}_{-} \cap \mathcal{A}_{+}$, if $L_{1} v=b,|b|_{Y} \leq K_{1}$, then $B(v) \leq K_{2}-\delta$ for some fixed $\delta>0$.

Let us solve $L_{1} v=b,|b|_{Y} \leq K_{1}, B(v)=d, d \in\left[K_{2}, K_{3}\right]$. Since $\operatorname{Im} L_{1}=$ $Y$, dim ker $L_{1}<\infty$, there is a right inverse $\widetilde{L}_{1}$ of $L_{1}$. We solve

$$
B\left(\widetilde{L}_{1} b+c v_{0}\right)=d .
$$

According to (H2-3) there are no solutions $c$ of (2.5) satisfying

$$
\widetilde{L}_{1} b+c v_{0} \in \mathcal{A}_{-} \cap \mathcal{A}_{+} .
$$

Hence (2.5) has precisely two solutions $c_{ \pm}=c_{ \pm}(b, d)$ such that

$$
\widetilde{L}_{1} b+c_{ \pm} v_{0} \in \mathcal{A}_{ \pm}, \quad \widetilde{L}_{1} b+c_{ \pm} v_{0} \notin \mathcal{A}_{+} \cap \mathcal{A}_{-} .
$$


Moreover, $B^{-1}\left(\left[K_{2}, K_{3}\right]\right)$ is a bounded subset. Hence $c_{ \pm}(\cdot, \cdot)$ is bounded as well. Summing up, we have

Proposition 2.5. If the hypotheses (H1-3) are satisfied, then the assumptions (a), (b) hold with $\mathcal{A}=\mathcal{A}_{ \pm}$.

Proof. (a) follows immediately, and (b) from Remark 2.1.

Applying Theorem 2.4 in the framework of Proposition 2.5, we can find at least two solutions for (2.1).

THEOREM 2.6. If the hypotheses (H1-3) are satisfied and $L_{2}$ is an isomorphism, then (2.1) has at least two solutions.

Pr o of. In this case, (2.4) has the form $u_{1}=\widetilde{L}_{2} V\left(u_{1}\right)$ and $V$ is bounded. Further, $V$ depends on $\mathcal{A}=\mathcal{A}_{ \pm}$. Applying the Schauder fixed point theory finishes the proof.

Now, assume in Theorem 2.4 that index $L_{2}=0$ and $\operatorname{dim} \operatorname{ker} L_{2}=1$. Then $S_{1} \simeq\{-1,1\}, H_{\infty}( \pm 1) \equiv H_{ \pm \infty}, D_{\infty}( \pm 1) \equiv D_{ \pm \infty}$ and $V_{\infty}( \pm 1) \equiv V_{ \pm \infty}$. Moreover, $V_{ \pm \infty}$ are uniquely determined by

$$
L_{1} V_{ \pm \infty}=H_{ \pm \infty}, \quad B\left(V_{ \pm \infty}\right)=D_{ \pm \infty}, \quad V_{ \pm \infty} \in \mathcal{A}
$$

The nontriviality of the stable homotopy class of $\eta$ means that $P V_{-\infty}$ and $P V_{+\infty}$ have opposite signs.

Note that the above arguments can be used for more general equations than (2.1). For instance, consider the following system of equations instead of $(2.2 \mathrm{a}, \mathrm{b})$ :

$$
\begin{aligned}
& L_{1} v=H(u), \\
& B(v)=D(u), \\
& L_{2} u=E(v),
\end{aligned}
$$

where $L_{1}, L_{2}, B, H, D$ have the properties (a), (b) and (P1-3), and $E$ : $Y \rightarrow Y$ is continuous. Then we deal with the map $\bar{\eta}=P E \circ V_{\infty} /\left|P E \circ V_{\infty}\right|_{Y}$ instead of $\eta$ in Theorem 2.4.

Finally, there is a special class of (2.1) which naturally satisfies (H2-3). Assume $\operatorname{Im} L_{1}=Y$, ker $L_{1}=\mathbb{R} v_{0}$, and $B(u)=\langle u, u\rangle$ for a symmetric, positive definite, continuous bilinear form $\langle\cdot, \cdot\rangle$ on $Y$. We see that in this case

$$
\begin{gathered}
\mathcal{A}_{+}=\left\{v \in X_{1} \mid\left\langle v, v_{0}\right\rangle \geq 0\right\}, \quad \mathcal{A}_{-}=\left\{v \in X_{1} \mid\left\langle v, v_{0}\right\rangle \leq 0\right\}, \\
\mathcal{A}_{-} \cap \mathcal{A}_{+}=\left\{v \in X_{1} \mid\left\langle v, v_{0}\right\rangle=0\right\} .
\end{gathered}
$$

Hence $\mathcal{A}_{-} \cap \mathcal{A}_{+} \oplus \mathbb{R} v_{0}$ is an orthogonal decomposition of $X_{1}$ with respect to $\langle\cdot, \cdot\rangle$.

Let us solve $L_{1} v=b,|b|_{Y} \leq K_{1}, v \in \mathcal{A}_{-} \cap \mathcal{A}_{+}$. This equation has the unique solution $v=L_{1}^{-1} b$, where $L_{1}^{-1}: Y \rightarrow \mathcal{A}_{-} \cap \mathcal{A}_{+}$is the inverse of $L_{1}$. Then 


$$
B(v)=\left\langle L_{1}^{-1} b, L_{1}^{-1} b\right\rangle \equiv \widetilde{B}(b) .
$$

Since $X_{1}$ is compactly embedded into $Y$ and $\langle\cdot, \cdot\rangle$ is continuous on $Y \times Y$, there is a smallest number $c_{L_{1}}$ such that

$$
\widetilde{B}(b) \leq c_{L_{1}}|b|_{Y}^{2}, \quad \forall b \in Y .
$$

Theorem 2.7. Assume $\operatorname{Im} L_{1}=Y$, $\operatorname{ker} L_{1}=\mathbb{R} v_{0}$, and $B(u)=\langle u, u\rangle$ for a symmetric, positive definite, continuous bilinear form $\langle\cdot, \cdot\rangle$ on $Y$. Let (H2) be satisfied. If

$$
c_{L_{1}} K_{1}^{2} \leq K_{2}-\delta
$$

for some fixed $\delta>0$, then (H3) holds as well.

Proof. The proof follows immediately by (2.8) and (2.9).

3. Applications. We return to (1.1) with $g(u)=G\left(\|u\|_{C^{0}}\right), G: \mathbb{R} \rightarrow \mathbb{R}$ continuous. We apply the results of Section 2 by putting

$$
\begin{aligned}
& X=\left\{z \in C^{3}([0,1], \mathbb{R}) \mid z(0)=z(1)=0\right\}, \quad Y=C^{0}([0,1], \mathbb{R}), \\
& X_{1}=C^{1}([0,1], \mathbb{R}), \quad X_{2}=\left\{z \in C^{2}([0,1], \mathbb{R}) \mid z(0)=z(1)=0\right\}, \\
& L u=u^{\prime \prime \prime}, \quad H(u)=f(u, \cdot), \\
& \bar{B}(u)=\left\|u^{\prime \prime}\right\|_{C^{0}}, \quad B(u)=\|u\|_{C^{0}}, \\
& L_{1} v=v^{\prime}, \quad L_{2} v=v^{\prime \prime}, \\
& D(u)=G\left(\|u\|_{C^{0}}\right) .
\end{aligned}
$$

First of all, we establish hypotheses (H1-3): (H1) is clear. (H2) is satisfied provided

$$
|f(\cdot, \cdot)| \leq K_{1}, \quad 0<K_{2} \leq G(\cdot) \leq K_{3} .
$$

In this case (see (H1)) $v_{0}(\cdot) \equiv 1$ and (see [2])

$$
\begin{gathered}
\mathcal{A}_{+}=\left\{v \in X_{1} \mid \max _{[0,1]} v \geq-\min _{[0,1]} v\right\}, \quad \mathcal{A}_{-}=\left\{v \in X_{1} \mid \max _{[0,1]} v \leq-\min _{[0,1]} v\right\}, \\
\mathcal{A}_{-} \cap \mathcal{A}_{+}=\left\{v \in X_{1} \mid \max _{[0,1]} v=-\min _{[0,1]} v\right\} .
\end{gathered}
$$

Hence (see [2]) $v \in \mathcal{A}_{-} \cap \mathcal{A}_{+}$implies

$$
\|v\|_{C^{0}}=\left(\max _{[0,1]} v-\min _{[0,1]} v\right) / 2 \leq \int_{0}^{1}\left|v^{\prime}(t)\right| d t / 2 \leq\left\|v^{\prime}\right\|_{C^{0}} / 2 .
$$

Thus, if $v \in \mathcal{A}_{-} \cap \mathcal{A}_{+}, v^{\prime}=b,\|b\|_{C^{0}} \leq K_{1}$ then

$$
B(v)=\|v\|_{C^{0}} \leq\left\|v^{\prime}\right\|_{C^{0}} / 2=\|b\|_{C^{0}} / 2 \leq K_{1} / 2 .
$$

Hence (H3) is satisfied if

$$
K_{1} / 2 \leq K_{2}-\delta, \quad \text { for a } \delta>0 .
$$


It is clear that $L_{2}$ is an isomorphism. Applying Theorem 2.6 we have

Theorem 3.1. Assume (3.1-2). Then (1.1) has at least two solutions.

Next we study

$$
\begin{aligned}
& v^{\prime}=f(u, t), \\
& \|v\|_{C^{0}}=G\left(\|u\|_{C^{0}}\right), \\
& u^{\prime}=e(v), \\
& u(0)=u(1),
\end{aligned}
$$

where $f, G$ are continuous satisfying (3.1-2), and $e: \mathbb{R} \rightarrow \mathbb{R}$ is continuous. We already know that (3.1-2) imply (a), (b) for $(2.7)$. Here $E(v)(t)=$ $e(v(t)), \forall t$, in the framework of (2.7).

Now we establish the conditions (P1-3) for this case by putting

$$
\begin{aligned}
& \lim _{r \rightarrow \pm \infty} f(r, t)=f_{ \pm}(t) \quad \text { uniformly in } t \in[0,1] \\
& f_{+}>0, f_{-}<0 \text { on }[0,1] \text { and } f_{ \pm} \text {are continuous, } \\
& \lim _{r \rightarrow \infty} G(r)=G_{\infty} .
\end{aligned}
$$

Since $L_{2} v=\left\{v^{\prime} \mid v(0)=v(1)\right\}$ in this case for (2.7), it is clear that $\operatorname{dim} \operatorname{ker} L_{2}=1$, $\operatorname{ker} L_{2}=\{v \equiv$ const $\}$, and $P v=\int_{0}^{1} v(t) d t$, where we identify constant functions with numbers.

Hence we can apply the ideas from the end of Section 2. We shall find the map $\bar{\eta}$ for this case. Here

$$
H_{ \pm \infty}=f_{ \pm}(\cdot), \quad D_{ \pm \infty}=G_{\infty} .
$$

We derive $V_{ \pm \infty}$ from $(2.6)$ :

$$
V_{+\infty}^{+}(t)=-\int_{t}^{1} f_{+}(s) d s+G_{\infty}, \quad V_{-\infty}^{+}(t)=\int_{0}^{t} f_{-}(s) d s+G_{\infty}
$$

for $\mathcal{A}=\mathcal{A}_{+}$, and

$$
V_{+\infty}^{-}(t)=\int_{0}^{t} f_{+}(s) d s-G_{\infty}, \quad V_{-\infty}^{-}(t)=-\int_{t}^{1} f_{-}(s) d s-G_{\infty}
$$

for $\mathcal{A}=\mathcal{A}_{-}$. We have used (3.2) and the inequalities $\left|f_{ \pm}(\cdot)\right| \leq K_{1}, G_{\infty} \geq K_{2}$.

Finally, we compute

and

$$
P E \circ V_{ \pm \infty}^{ \pm}=\int_{0}^{1} e\left(-\int_{t}^{1} f_{ \pm}(s) d s \pm G_{\infty}\right) d t
$$

$$
P E \circ V_{ \pm \infty}^{\mp}=\int_{0}^{1} e\left(\int_{0}^{t} f_{ \pm}(s) d s \mp G_{\infty}\right) d t .
$$


Summing up, we obtain

THEOREM 3.2. Assume (3.1-3). If

$$
\begin{aligned}
& \int_{0}^{1} e\left(-\int_{t}^{1} f_{+}(s) d s+G_{\infty}\right) d t \cdot \int_{0}^{1} e\left(\int_{0}^{t} f_{-}(s) d s+G_{\infty}\right) d t<0, \\
& \int_{0}^{1} e\left(\int_{0}^{t} f_{+}(s) d s-G_{\infty}\right) d t \cdot \int_{0}^{1} e\left(-\int_{t}^{1} f_{-}(s) d s-G_{\infty}\right) d t<0
\end{aligned}
$$

then (3.4) has at least two solutions. Moreover, if at least one of these inequalities holds, then (3.4) has a solution.

Proof. The proof follows immediately from the note at the end of Section 2 pertinent to (2.7) provided that we take either $\mathcal{A}=\mathcal{A}_{+}$or $\mathcal{A}=\mathcal{A}_{-}$. Indeed, according to the above derivation we have

$$
\begin{aligned}
& \bar{\eta}(+1) / \int_{0}^{1} e\left(-\int_{t}^{1} f_{+}(s) d s+G_{\infty}\right) d t>0, \\
& \bar{\eta}(-1) / \int_{0}^{1} e\left(\int_{0}^{t} f_{-}(s) d s+G_{\infty}\right) d t>0
\end{aligned}
$$

for $\mathcal{A}=\mathcal{A}_{+}$, and

$$
\begin{aligned}
& \bar{\eta}(+1) / \int_{0}^{1} e\left(\int_{0}^{t} f_{+}(s) d s-G_{\infty}\right) d t>0 \\
& \bar{\eta}(-1) / \int_{0}^{1} e\left(-\int_{t}^{1} f_{-}(s) d s-G_{\infty}\right) d t>0
\end{aligned}
$$

for $\mathcal{A}=\mathcal{A}_{-}$. The assumptions of Theorem 3.2 express the nontriviality of the stable homotopy class of $\bar{\eta}$ for $\mathcal{A}=\mathcal{A}_{+}$and $\mathcal{A}=\mathcal{A}_{-}$, respectively.

To apply Theorem 2.7 , let us consider

$$
\begin{aligned}
& u^{\prime \prime \prime}=f(u, t), \\
& \int_{0}^{1}\left(u^{\prime \prime}(t)\right)^{2} d t=G\left(\|u\|_{C^{0}}\right), \\
& u(0)=u(1)=0,
\end{aligned}
$$

where $f, G$ are continuous and satisfy (3.1).

THEOREM 3.3. If

$$
K_{1}^{2} / \pi^{2} \leq K_{2}-\delta \quad \text { for some fixed } \delta>0,
$$

then (3.5) has at least two solutions. 
Proof. We apply Theorems 2.6 and 2.7. For this purpose, we have to verify (2.9). We have

$$
L_{1} v=v^{\prime}, \quad\langle u, v\rangle=\int_{0}^{1} u(t) v(t) d t
$$

in the framework of Theorem 3.1 for this case. So we obtain

$$
L_{1} v=b, v \in \mathcal{A}_{-} \cap \mathcal{A}_{+} \Leftrightarrow v^{\prime}=b, \int_{0}^{1} v(t) d t=0 .
$$

Note that the sets $\mathcal{A}_{-}, \mathcal{A}_{+}, \mathcal{A}_{-} \cap \mathcal{A}_{+}$are defined by the formulas preceding Theorem 2.7.

Hence (see (2.8))

$$
\widetilde{B}(b)=\int_{0}^{1} v(t)^{2} d t=\int_{0}^{1} w^{\prime}(t)^{2} d t=\int_{0}^{1} b(t) w(t) d t,
$$

where

$$
w(t) \equiv-\int_{0}^{t} v(s) d s, \quad w^{\prime \prime}=-b, \quad w(0)=w(1)=0 .
$$

On the other hand, by Wirtinger's inequality we have for any $u \in C^{2}$ with $u(0)=u(1)=0$,

$$
\pi^{2}\|u\|_{L^{2}}^{2}=\pi^{2} \int_{0}^{1} u(t)^{2} d t \leq-\int_{0}^{1} u^{\prime \prime}(t) u(t) d t \leq\left\|u^{\prime \prime}\right\|_{L^{2}}\|u\|_{L^{2}},
$$

and so $\pi^{2}\|u\|_{L^{2}} \leq\left\|u^{\prime \prime}\right\|_{L^{2}}$. Thus

$$
\widetilde{B}(b)=\int_{0}^{1} b(t) w(t) d t \leq\|w\|_{L^{2}}\|b\|_{L^{2}} \leq \frac{1}{\pi^{2}}\|b\|_{C^{0}}^{2} .
$$

This implies $c_{L_{1}} \leq 1 / \pi^{2}$. The proof is finished, since (3.6) implies (2.9).

\section{Concluding remarks}

Remark 4.1. First of all, we show that the validity of the assumptions of Theorem 3.2 strongly depends on the choice of the function $e$. If $e(z) \equiv z$ then (3.4) is a second-order differential equation

$$
\begin{aligned}
& u^{\prime \prime}=f(u, t), \\
& \left\|u^{\prime}\right\|_{C^{0}}=G\left(\|u\|_{C^{0}}\right), \\
& u(0)=u(1),
\end{aligned}
$$

and (2.7) is precisely $(2.2 \mathrm{a}, \mathrm{b})$. The assumptions of Theorem 3.2 are never satisfied for this case. This follows easily from $\left|f_{ \pm}(\cdot)\right| \leq K_{1}, G_{\infty} \geq K_{2}$ and $2 K_{2}>K_{1}$, since the integral inequalities are not satisfied. 
On the other hand, it is also not hard to verify that the conditions (3.1-2) for the case $e(z) \equiv z$ in (3.4) imply the nonexistence of solutions for (3.4). Indeed, if $v$ is a solution of (3.4) for this case, then

$$
\begin{aligned}
& \left\|v^{\prime}\right\|_{C^{0}} \leq K_{1}, \quad\|v\|_{C^{0}} \geq K_{2}, \\
& \int_{0}^{1} v(t) d t=0, \quad v \in \mathcal{A}_{+} \cup \mathcal{A}_{-} .
\end{aligned}
$$

Assume $v \in \mathcal{A}_{+}$(the case $v \in \mathcal{A}_{-}$is similar). Hence

$$
\max _{[0,1]} v=v\left(t_{\max }\right)=\|v\|_{C^{0}} \geq K_{2}
$$

and $v(t)=v\left(t_{\max }\right)+\int_{t_{\max }}^{t} v^{\prime}(s) d s$. Thus

$$
0=\int_{0}^{1} v(t) d t \geq K_{2}-\int_{0}^{1} \int_{0}^{t} K_{1} d s d t=K_{2}-\frac{K_{1}}{2}>0 \quad(\text { by }(3.2)) .
$$

This is a contradiction, proving the nonexistence of solutions for (4.1) under the conditions $(3.1-2)$.

Remark 4.2. Of course, the results of Section 2 suggest a broader variety of equations than (1.1), (3.4) and (3.5). The derivation of assumptions (H1-3), (P1-3) is similar, but it is generally more complicated. We have chosen the above simple examples as an illustration for possible applications of our method. Moreover, the hypotheses $(\mathrm{H} 1-3)$ can be generalized to the case $\infty>\operatorname{dim} \operatorname{ker} L_{1}>1, \operatorname{Im} L_{1}=Y$. For instance, consider the following system of equations:

$$
\begin{aligned}
& u_{1}^{\prime \prime \prime}=f_{1}\left(u_{1}, \ldots, u_{k}, t\right), \\
& \vdots \\
& u_{k}^{\prime \prime \prime}=f_{k}\left(u_{1}, \ldots, u_{k}, t\right), \\
& \left\|u_{1}^{\prime \prime}\right\|_{C^{0}}=G_{1}\left(\left\|u_{1}\right\|_{C^{0}}, \ldots,\left\|u_{k}\right\|_{C^{0}}\right), \\
& \vdots \\
& \left\|u_{k}^{\prime \prime}\right\|_{C^{0}}=G_{k}\left(\left\|u_{1}\right\|_{C^{0}}, \ldots,\left\|u_{k}\right\|_{C^{0}}\right), \\
& u_{1}(0)=u_{1}(1)=\ldots=u_{k}(0)=u_{k}(1)=0,
\end{aligned}
$$

where $f_{1}, \ldots, f_{k}: \mathbb{R}^{k} \times[0,1] \rightarrow \mathbb{R}$ and $G_{1}, \ldots, G_{k}: \mathbb{R}^{k} \rightarrow \mathbb{R}$ are continuous and satisfy

$$
\left|f_{i}\right| \leq K_{1 i}, \quad 0<K_{2 i} \leq G_{i} \leq K_{3 i}, \quad i=1, \ldots, k,
$$

for some constants $K_{j i}, i=1, \ldots, k, j=1,2,3$, such that 


$$
K_{1 i} / 2 \leq K_{2 i}-\delta, \quad i=1, \ldots, k,
$$

for some fixed $\delta>0$.

Then applying the procedure of Section 3 (see the arguments before Theorem 3.1), we conclude that (4.2) has at least $2^{k}$ solutions. Note that in this case $L_{1}\left(u_{1}, \ldots, u_{k}\right)=\left(u_{1}^{\prime}, \ldots, u_{k}^{\prime}\right)$ and $\operatorname{dim} \operatorname{ker} L_{1}=k$.

Remark 4.3. If the equation (3.4) depends in some way on a parameter in Theorem 3.2, then we may find three domains of the parameter for which, respectively, this equation has no, at least one and at least two solutions. This remark holds generally. To be more concrete, we return to the problem (4.1) from Remark 4.1. We embed this problem in the following family of equations:

$$
\begin{aligned}
& u^{\prime \prime}=f(u, t), \\
& \left\|u^{\prime}+\lambda\right\|_{C^{0}}=G\left(\|u\|_{C^{0}}\right), \\
& u(0)=u(1),
\end{aligned}
$$

where $\lambda \in \mathbb{R}$ is a parameter. We assume the validity of (3.1-3). By putting $e(v)=v-\lambda$, we can apply the method of Section 3 used for (3.4). Conditions similar to those of Theorem 3.2 read as follows:

$$
\begin{aligned}
& \left(-\int_{0}^{1} \int_{t}^{1} f_{+}(s) d s d t+G_{\infty}-\lambda\right) \cdot\left(\int_{0}^{1} \int_{0}^{t} f_{-}(s) d s d t+G_{\infty}-\lambda\right)<0, \\
& \left(\int_{0}^{1} \int_{0}^{t} f_{+}(s) d s d t-G_{\infty}-\lambda\right) \cdot\left(-\int_{0}^{1} \int_{t}^{1} f_{-}(s) d s d t-G_{\infty}-\lambda\right)<0 .
\end{aligned}
$$

Assume $f_{ \pm}(s) \equiv f_{ \pm}$for some constants $0>f_{-}>-f_{+}$. Then (4.4) has the form

$$
\begin{gathered}
G_{\infty}-f_{+} / 2<\lambda<G_{\infty}+f_{-} / 2, \\
-G_{\infty}-f_{-} / 2<\lambda<-G_{\infty}+f_{+} / 2 .
\end{gathered}
$$

Note that $G_{\infty}>\left|f_{ \pm}\right| / 2$, since (3.1-3) hold. Hence

$$
f_{+} / 2-G_{\infty}<0<G_{\infty}-f_{+} / 2 .
$$

On the other hand, using the same arguments as in Remark 4.1 we see that if (4.3) has a solution then

$$
K_{2}-K_{1} / 2 \leq|\lambda| \leq K_{3}+K_{1} / 2 .
$$

Indeed, the difference between this case and the one in Remark 4.1 is only the relation

$$
\int_{0}^{1} v(t) d t=\lambda
$$

since $v=u^{\prime}+\lambda$ for this case. 
Summarizing, we obtain:

1. If (4.7) does not hold then (4.3) has no solution.

2. If either (4.5) or (4.6) holds then (4.3) has at least one solution.

Of course, the result of Remark 4.1 is contained in the above statement.

\section{References}

[1] M. S. Berger, Nonlinearity and Functional Analysis, Academic Press, New York, 1977.

[2] S. A. Brykalov, The solvability of a nonlinear boundary value problem in a fixed set of functions, Differentsial'nye Uravneniya 27 (1991), 2027-2033 (in Russian).

MATHEMATICAL INSTITUTE

SLOVAK ACADEMY OF SCIENCES

ŠTEFÁNIKOVA 49

81473 BRATISLAVA, SLOVAKIA

Reçu par la Rédaction le 15.12.1992

Révisé le 3.3 .1993 